\title{
Multiuser diversity in correlated Rayleigh-fading channels
}

\author{
Behrooz Makki and Thomas Eriksson
}

\begin{abstract}
This article studies the effect of scheduling and multiuser diversity on the performance of correlated Rayleighfading channels. More specifically, the power-limited channel average rate is obtained for quasi-static correlated fading channels. The results are obtained in the cases where there is perfect or imperfect channel state information available at the transmitter. Simulation results show that the average rate reduction due to channels dependencies is ignorable in low correlation conditions. However, the effect of scheduling and multiuser diversity on the average rate reduces substantially as the fading channels dependency increases. Also, for different channels correlation conditions, considerable performance improvement is achieved via very limited number of feedback bits.
\end{abstract}

\section{Introduction}

Employment of adaptive modulation and scheduling leads to substantial performance improvement in multiuser systems, normally called multiuser diversity [1-12]. This is the main motivation for the current schedulingbased systems and this article as well. In these methods, the transmitter is provided with some information about the channel quality of different users. This information is then utilized by a scheduler to select the appropriate users, coding, and modulation such that an objective function is optimized. System throughput and fairness between the users are two objective functions mainly considered in the literature. Furthermore, depending on the number of users, channels characteristics and the feedback load resources, the transmitter information about the channels quality can be perfect or imperfect.

Assuming different levels of channel state information (CSI), a large number of scientific reports can be found that have tackled the multiuser diversity problem in different theoretical and practical aspects. For instance, [6-12] investigated the performance of multiuser networks under perfect CSI assumption. These works were later extended by, e.g., [13-19] where the system performance was analyzed in the presence of imperfect CSI available at the scheduler. Furthermore, among different research projects involving in this topic the WINNER+ [20] and the 3rd Generation Partnership Project (3GPP) [21] can be

\footnotetext{
* Correspondence: behrooz.makki@chalmers.se

Chalmers University of Technology, Gothenburg, Sweden
}

mentioned where multiuser diversity is one of the most important issues.

References [6-19] are all based on the assumption that the fading channels are mutually independent. That is, the network performance is investigated in the case where there is no correlation between the fading channels of different transmission end-points. However, based on the environmental properties, realistic channels may not be independent [3-5], [22-24]. Therefore, it is important to study the channel performance under correlated channels condition.

In this perspective, this article studies the average rate of correlated Rayleigh-fading multiuser networks. The results are obtained for quasi-static channels in the cases where there is perfect or imperfect CSI available at the transmitter. It is mainly focused on a system with a single transmitter and two receivers, which allows us to find closed-form solutions for the average rate and power allocation criteria. However, some discussions about extending the results to arbitrary number of receivers are also presented and the final conclusions are valid independent of the number of receivers. Assuming imperfect CSI, we evaluate the effect of optimal channel quantization on the system performance. The results show that substantial performance improvement is achieved with a limited number of feedback bits per user. Moreover, the effect of scheduling and multiuser diversity reduces with the channels correlation, although the rate reduction is ignorable in low correlation conditions. The arguments would be interesting for people involved in WINNER+, 
3GPP or the ones working on scheduling between close users, for instance scheduling in single-cell networks, e.g., [25-28].

\section{System model}

In this part, we consider a network with a single transmitter and two receivers equipped with a single antenna. In time slot $t$, a max-rate scheduler selects one of the receivers, e.g., the $k$-th receiver. Then, the length- $L_{\mathrm{c}}$ codeword $\left\{X_{t}[i] \mid i=1, \ldots, L_{\mathrm{c}}\right\}$ multiplied by the random variable $H_{k, t}$ is summed with independent and identically distributed (i.i.d) complex Gaussian noise ${ }^{\mathrm{a}}$ samples $\left\{Z_{k, t}[i] \mid i=1, \ldots, L_{c}, Z_{k, t}[i] \sim \mathcal{C N}\left(0, \sigma_{k}^{2}\right)\right\}$ resulting in

$$
Y_{k, t}[i]=H_{k, t} X_{t}[i]+Z_{k, t}[i], \quad i=1, \ldots, L_{\mathrm{c}} .
$$

For simplicity of notation, the time slot index $t$ is dropped. A quasi-static correlated Rayleigh-fading channel model is considered $^{\mathrm{b}}$; The channel gains $G_{k} \doteq\left|H_{k}\right|^{2}, k=1,2$, remain constant for a long time and then change according to their corresponding joint fading probability density function (pdf) $f_{G_{1}, G_{2}}(x, y)$. Also, the gains are supposed to have identical marginal pdfs $f_{G_{k}}(x)=\frac{1}{\mu} e^{-}-\frac{x}{\mu}, x \geq 0, k=1,2$, and the relation between the fading variables is modeled by

$$
H_{1}=\beta H_{2}+\sqrt{1-\beta^{2}} \varepsilon, \quad \varepsilon: \mathcal{C N}(0, \mu) .
$$

Here, $\mu$ denotes the exponential pdf parameter determined by the path loss and shadowing between the terminals and $\beta$ is a known correlation factor modeling the two variables dependencies. This is a well-established model considered in the literature for different phenomena such as CSI imperfection, estimation error and channels/signals correlation [29-32]. In this way, the joint pdf of the gains is found as

$$
f_{G_{1}, G_{2}}(x, y)=\frac{1}{\left(1-\beta^{2}\right) \mu^{2}} e^{-\lambda \frac{x+y}{\left(1-\beta^{2}\right) \mu}} I_{0}\left(\frac{2 \beta \sqrt{x y}}{\left(1-\beta^{2}\right) \mu}\right)
$$

where $I_{0}($.$) is the zeroth-order modified Bessel func-$ tion of the first kind [33]. Finally, note that as the channels have identical pdfs, the max-rate scheduler which transmits to the user with the strongest channel at any given time slot not only optimizes the system total performance but also maintains the long-term fairness between the users. Moreover, although it is the simplest to assume a network with two users, as seen in the following, the results provide valuable insights for the more general cases with arbitrary number of users. Also, extension of the results to arbitrary number of receivers experiencing different fading distributions can be found in the Appendix.
It is assumed that each receiver has perfect CSI about its corresponding channel gain which is an acceptable assumption in quasi-static condition, e.g., [17,18,34-38]. However, the transmitter may be provided with imperfect (Section 3) or perfect (Section 4) CSI about the fading channels. Further, all results are presented in natural logarithm basis, the channel average rate is presented in nats-per-channel-use (npcu) and, as stated in the following, the arguments are restricted to Gaussian input distributions. Finally, note that Rayleigh-fading channels are good models for tropospheric and ionospheric signal propagation as well as the effect of heavily built-up urban environments on radio signals $[39,40]$. Also, it is most applicable when there is no dominant propagation along a line of sight between the transmitters and the receivers.

\subsection{Average rate with no CSI at the transmitter}

As a system performance lower bound, it is interesting to study the channel average rate with no CSI at the transmitter. In this case, the channel average rate is simplified to the one for a single user network, as one of the users is selected by the scheduler randomly. Also, with no CSI at the transmitter, the data is transmitted at a fixed rate $R$ which is decoded if the channel realization supports the rate, i.e., $R \leq \log (1+g T)$ where $T$ is the transmission power. ${ }^{\mathrm{c}}$ Therefore, representing the gains cumulative distribution function (cdf) by $F_{G}(g)$, the no-CSI channel average rate is obtained by

$$
\bar{R}_{\mathrm{no}}=\max _{R} R\left(1-F_{G}\left(\frac{e^{R}-1}{T}\right)\right)
$$

which for Rayleigh-fading gain distribution results in

$$
\bar{R}_{\mathrm{no}}=\ell_{W}(\mu T) e^{-\frac{e^{\ell} W(\mu T)_{-1}}{T}} .
$$

Here, $\ell_{W}(x)$ is the standard Lambert $W$ function defined as

$$
x e^{x}=y \Rightarrow x=\ell_{W}(y) .
$$

\section{Average rate in the presence of imperfect CSI at the transmitter}

This section studies the channel average rate in the case where the scheduler is provided with quantized CSI about the fading channels. In this way, considering $N$ quantization regions, the quantization encoder function

$$
C\left(g_{k}\right)=i \text { if } g_{k} \in S_{i}=\left[\tilde{g}_{i-1}, \tilde{g}_{i}\right), \tilde{g}_{0}=0, \tilde{g}_{N}=\infty
$$

is implemented by each receiver. Here, $\tilde{g}_{i}$ 's denote the quantization boundaries and $S_{i}$ is the $i$-th 
quantization region. The quantization indices are sent back to the scheduler which selects the user with the higher quantization index (max-rate scheduler). Also, if the channel gains are in the same quantization regions, one of them is selected randomly.

Remark 1: The optimal max-rate scheduler should select the users with the highest SNR. However, as stated in the following, the water-filling properties imply that higher powers are allocated to the higher quantization regions (see, e.g., (14), (19) and [34-38]). Therefore, the SNR increases with the quantization index and scheduling based on the quantization indices works the same as scheduling based on the SNRs.

Provided that the scheduled user channel gain is in the region $S_{i}$, a fixed gain $\hat{g}_{i} \in\left[\tilde{g}_{i-1}, \tilde{g}_{i}\right)$ is considered by the transmitter and the data is sent with power $T_{i}$ and rate $R_{i}=\log \left(1+\hat{g}_{i} T_{i}\right)$. The data is successfully decoded at the corresponding receiver if $G_{k} \geq \hat{g}_{i}$ where $k$ represents the selected user index. Therefore, considering all quantization regions, the channel average rate is found as

$$
\bar{R}=2 \sum_{i=2}^{N} P_{i} R_{i}+\sum_{i=1}^{N} Q_{i} R_{i} .
$$

Here,

$$
\begin{aligned}
P_{i} & =\operatorname{Pr}\left\{G_{1} \in\left[\hat{g}_{i}, \tilde{g}_{i}\right) \& G_{2} \in\left[0, \tilde{g}_{i-1}\right)\right\} \\
& =\int_{\hat{g}_{i}}^{\tilde{g}_{i}} \int_{0}^{\tilde{g}_{i-1}} f_{G_{1}, G_{2}}(x, y) \mathrm{d} x \mathrm{~d} y
\end{aligned}
$$

is the probability that (1) for instance, channel $G_{1}$ is in the $i$-th quantization region, (2) its corresponding channel gain is higher than the considered value $\hat{g}_{i}$, that is, $G_{1} \in\left[\hat{g}_{i}, \tilde{g}_{i}\right)$ and (3) the second user channel gain is in one of the quantization regions $S_{j}, j<i$, such that the first user is selected by the scheduler. Then, the first summation term is multiplied by two, as the same thing can happen for the other user. Furthermore, $Q_{i}$ is found as

$$
\begin{aligned}
Q_{i} & =\operatorname{Pr}\left\{G_{1} \in\left[\hat{g}_{i}, \tilde{g}_{i}\right) \& G_{2} \in\left[\tilde{g}_{i-1}, \tilde{g}_{i}\right)\right\} \\
& =\int_{\hat{g}_{i}}^{\tilde{g}_{i}} \int_{\tilde{g}_{i-1}}^{\tilde{g}_{i}} f_{G_{1}, G_{2}}(x, y) \mathrm{d} x \mathrm{~d} y
\end{aligned}
$$

which is the probability that (1) both users are in the $i$-th quantization region and (2) the channel gain of the selected user supports the rate, e.g., $G_{1} \in\left[\hat{g}_{i}, \tilde{g}_{i}\right)$ if the first user is selected by the scheduler. Note that in this case one of the users is scheduled randomly with probability $\frac{1}{2}$. Therefore, the second summation term in (7) is not multiplied by two. Correspondingly, the average transmission power is obtained by

$$
\bar{T}=2 \sum_{i=2}^{N} P^{\prime}{ }_{i} T_{i}+\sum_{i=1}^{N} Q_{i}^{\prime} T_{i}
$$

where

$$
\begin{aligned}
P^{\prime}{ }_{i} & =\operatorname{Pr}\left\{G_{1} \in S_{i} \& G_{2} \in S_{j}, j<i\right\} \\
& =\int_{\tilde{g}_{i-1}}^{\tilde{g}_{i}} \int_{0}^{\tilde{g}_{i-1}} f_{G_{1}, G_{2}}(x, y) \mathrm{d} x \mathrm{~d} y
\end{aligned}
$$

denotes the probability that, for instance, channel $G_{1}$ is in the $i$-th quantization region while the second user channel gain is in one of the lower regions. Also,

$$
\begin{aligned}
Q_{i}^{\prime} & =\operatorname{Pr}\left\{G_{1} \in S_{i} \& G_{2} \in S_{i}\right\} \\
& =\int_{\tilde{g}_{i-1}}^{\tilde{g}_{i}} \int_{\tilde{g}_{i-1}}^{\tilde{g}_{i}} f_{G_{1}, G_{2}}(x, y) \mathrm{d} x \mathrm{~d} y
\end{aligned}
$$

is the probability that both channels are in the $i$-th quantization region where one of them is selected randomly.

Using (7), (10) and the power constraint $\bar{T} \leq T$, the power-limited average rate maximization problem can be stated as

$$
\begin{aligned}
& \max _{\tilde{g}_{i} \hat{g}_{i} T_{i}}\left\{2 \sum_{i=2}^{N} P_{i} R_{i}+\sum_{i=1}^{N} Q_{i} R_{i}\right\} \\
& \text { subject to }\left\{2 \sum_{i=2}^{N} P^{\prime}{ }_{i} T_{i}+\sum_{i=1}^{N} Q_{i}^{\prime} T_{i} \leq T\right\}
\end{aligned}
$$

which, as discussed in [34-38], [[41], Section 9.4], is a convex problem in terms of transmission powers $T_{i}$. Therefore, the optimal transmission powers can be determined based on the Lagrange multiplier function $\Upsilon=\bar{R}-\lambda \bar{T}$ which leads to the water-filling equations

$$
\frac{\partial \Upsilon}{\partial T_{i}}=0 \Rightarrow\left\{\begin{array}{l}
T_{1}=\left\lceil\frac{Q_{1}}{\lambda Q_{1}}-\frac{1}{\hat{\mathrm{g}}_{1}}\right]^{+}, i=1 \\
T_{i}=\left\lceil\frac{2 P_{i}+Q_{i}}{\lambda\left(2 P^{\prime}{ }_{i}+Q_{i}^{\prime}\right)}-\frac{1}{\hat{8}_{1}}\right]^{+}, i>1
\end{array} .\right.
$$

Here, $\lambda$ is the Lagrange multiplier satisfying $\bar{T} \leq T$ and $\lceil x\rceil^{+} \doteq \max (0, x)$. Intuitively, using optimal power allocation the power is not wasted on weak channel realization and the saved power is spent on strong gain realizations. Therefore, there will be a quantization index $\hat{i}$ where $T_{i}=0$ if $i<\hat{i}$ and $T_{i}>0$ if $i \geq \hat{i}$. This point is 
helpful for simplifying the water-filling power allocation algorithm.

Considering (13), the main problem is to find the probability terms in (7) and (10) which can be found according to the following procedure

$$
\begin{aligned}
& \int_{u}^{v} \int_{w}^{z} f_{\mathrm{G}_{1}, \mathrm{G}_{2}}(x, y) \mathrm{d} \mathrm{d} \mathrm{d} y \\
& \stackrel{(a)}{=} \int_{u}^{v} \frac{1}{\mu} e^{-\frac{x}{r}}\left(\begin{array}{l}
\sqrt{\frac{2 z}{r}} \\
\frac{\sqrt{\frac{2 w}{r}}}{r}
\end{array} e^{-\frac{\theta^{2}}{2}} I_{0}(s \sqrt{x} \theta) \mathrm{d} \theta\right) \mathrm{d} x \\
& \underline{\underline{(b)}} \int_{u}^{v} \frac{1}{\mu} e^{-\frac{x}{\mu}}\left\{\xi\left(s \sqrt{x}, \sqrt{\frac{2 w}{r}}\right)-\xi\left(s \sqrt{x}, \sqrt{\frac{2 z}{r}}\right)\right\} \mathrm{d} x \\
& \underline{\underline{(c)}}\left(1-\beta^{2}\right) e^{-\frac{w}{\mu}}\left\{\xi\left(\sqrt{\frac{2 w}{r}} \beta, \sqrt{\frac{2 u}{r}}\right)-\xi\left(\sqrt{\frac{2 w}{r}} \beta, \sqrt{\frac{2 v}{r}}\right)\right\} \\
& -\left(1-\beta^{2}\right) e^{-\frac{z}{\mu}}\left\{\xi\left(\sqrt{\frac{2 z}{r}} \beta, \sqrt{\frac{2 u}{r}}\right)-\xi\left(\sqrt{\frac{2 z}{r}} \beta, \sqrt{\frac{2 v}{r}}\right)\right\} \\
& +\frac{1}{\mu} \int_{u}^{v} e^{-\frac{x}{\mu}}\left\{\xi\left(\sqrt{\frac{2 z}{r}}, s \sqrt{x}\right)-\xi\left(\sqrt{\frac{2 w}{r}}, s \sqrt{x}\right)\right\} \mathrm{d} x \\
& \underline{\underline{(d)}} e^{-\frac{w}{\mu}}\left\{\phi\left(w \beta^{2}, u\right)-\phi\left(w \beta^{2}, v\right)\right\}-e^{-\frac{z}{\mu}}\left\{\phi\left(z \beta^{2}, u\right)-\phi\left(z \beta^{2}, v\right)\right\} \\
& +e^{-\frac{v}{\mu}} \phi\left(w, v \beta^{2}\right)-e^{-\frac{u}{\mu}} \phi\left(w, u \beta^{2}\right)-e^{-\frac{v}{\mu}} \phi\left(z, v \beta^{2}\right)+e^{-\frac{u}{\mu}} \phi\left(z, u \beta^{2}\right) .
\end{aligned}
$$

Here, $(a)$ is obtained by defining $r \doteq\left(1-\beta^{2}\right) \mu, s \doteq \sqrt{2 / r} \beta$ and using variable transform $\theta=\sqrt{2 \gamma / r}$. Then, $(b)$ is directly obtained from the definition of the Marcum Qfunction

$$
\xi(x, y)=\int_{\gamma}^{\infty} t e^{-\frac{t^{2}+x^{2}}{2}} I_{0}(x t) \mathrm{d} t .
$$

Also, $(c)$ is based on the fact that

$$
\xi(x, y)=1+e^{-\left(x^{2}+y^{2}\right) / 2} I_{0}(x y)-\xi(y, x)
$$

and finally, $(d)$ is derived by using variable transform $t=\sqrt{x} \quad, \quad$ partial integration, defining $\phi(x, y) \doteq \xi\left(\sqrt{\frac{2 x}{r}}, \sqrt{\frac{2 y}{r}}\right)$ and some calculations.

A simple average rate optimization algorithm: In contrast to transmission power parameters, the power-limited average rate optimization problem of quantized CSI-based systems, e.g., (13), is not a convex optimization problem in terms of quantization parameters $\hat{g}_{i}, \tilde{g}_{i} \forall_{i}$ [34-38]. Therefore, although implementable, gradient-based algorithms are not efficient in determining the optimal quantization parameters. In order to tackle this problem, we propose an iterative algorithm, illustrated in Algorithm 1.

Remark 2: Similar to other techniques for solving nonconvex optimization problems, it can not be guaranteed that the algorithm leads to the globally optimal solution for all channel conditions. However, by extensive testing, it is observed that for many different initial parameter settings and vector generation procedures, the algorithm leads to unique solutions. Furthermore, our experiments show that the algorithm is much more efficient than using greedy search scheme which requires a large number of initial random seeds due to the non-convexity of (13). Finally, although it may be time-consuming when the number of optimization parameters increases, the proposed algorithm has been shown to be efficient in many complex optimization problems dealing with local minima issues [42].

In the following, the channel average rate in the presence of perfect CSI available at the transmitter is studied and then the simulation results are presented in Section 5.

\section{Average rate in the presence of perfect CSI at the transmitter}

Assuming perfect CSI at the transmitter, the data is always transmitted to the user with higher instantaneous channel gain. Therefore, the channel average rate is rephrased as

$$
\bar{R}=\int_{0}^{\infty} f_{Z}(z) \log (1+z T(z)) \mathrm{d} z
$$

where $Z \doteq \max \left(G_{1}, G_{2}\right)$ is an auxiliary variable with pdf $f_{Z}(z)$ and $T(z)$ is the transmission power considered for the instantaneous variable realization $Z=z$. Then, the average transmission power is obtained by $\bar{T}=\int_{0}^{\infty} T(z) f_{Z}(z) \mathrm{d} z$. Therefore, using the Lagrange multiplier function $\Upsilon=\bar{R}-\lambda \bar{T}$, the optimal power allocation is found as

$$
\frac{\partial \Upsilon}{\partial T(z)}=0 \Rightarrow T(z)=\left\{\begin{array}{c}
\frac{1}{\lambda^{*}}-\frac{1}{z}, z \geq \lambda^{*} \\
0, \quad z<\lambda^{*}
\end{array}\right.
$$

Here, the water-filling threshold $\lambda^{\prime \prime}$ determined according to

$$
\begin{gathered}
\lambda^{*}=\underset{\lambda}{\arg }\left\{\int_{\lambda}^{\infty}\left(\frac{1}{\lambda}-\frac{1}{z}\right) f_{Z}(z) \mathrm{d} z=T\right\} \\
\stackrel{(e))}{=} \underset{\lambda}{\arg }\left\{\int_{\lambda}^{\infty} \frac{1-F_{Z}(z)}{z^{2}} \mathrm{~d} z=T\right\}
\end{gathered}
$$

where $F_{Z}(z)$ is the cdf of the variable $Z$ and $(e)$ is obtained by partial integration. Finally, from (18) and (19), the channel average rate can be rephrased as

$$
\begin{aligned}
\bar{R} & =\int_{\lambda^{*}}^{\infty} f_{Z}(z) \log (1+z T(z)) \mathrm{d} z \\
& =\int_{\lambda^{*}}^{\infty} f_{Z}(z) \log \left(\frac{z}{\lambda^{*}}\right) \mathrm{d} z \underline{\underline{(f)}} \int_{\lambda^{*}}^{\infty} \frac{1-F_{Z}(z)}{z} \mathrm{~d} z
\end{aligned}
$$


where $(f)$ is again based on partial integration. Therefore, the main problem would be to determine the cdf $F_{Z}(z),(20)$ and then (21).

Considering (15), the auxiliary variable cdf is found as

$$
\begin{aligned}
F_{Z}(z) & =\operatorname{Pr}\left\{\max \left(G_{1}, G_{2}\right) \leq z\right\} \\
& =\operatorname{Pr}\left\{G_{1} \leq z \& G_{2} \leq z\right\}=\int_{0}^{z} \int_{0}^{z} f G_{1}, G_{2}(x, y) \mathrm{d} x \mathrm{~d} y \\
& \underline{\underline{(g)}} 1-e^{-\frac{z}{\mu}}\left\{1-\xi\left(\sqrt{\frac{2 z}{r}} \beta, \sqrt{\frac{2 z}{r}}\right)+\xi\left(\sqrt{\frac{2 z}{r}}, \sqrt{\frac{2 z}{r}} \beta\right)\right\}
\end{aligned}
$$

where $(g)$ is based on the fact that $\xi(x, 0)=1, \xi(0, x)=e^{-\frac{x^{2}}{2}}$ and $r=\left(1-\beta^{2}\right) \mu$. Moreover, it can be written

$$
\begin{aligned}
& J(z)=1-F_{Z}(z) \\
& \underline{\underline{(h h}} e^{-\frac{z}{\mu}}\left\{1+2 \xi\left(\sqrt{\frac{2 z}{r}}, \sqrt{\frac{2 z}{r}} \beta\right)-e^{-\frac{z}{r}\left(1+\beta^{2}\right)} I_{0}\left(\frac{2 z}{r} \beta\right)\right\} \\
& \underline{\underline{(i)}} 2 e^{-\frac{z}{\mu}}-2 e^{-\frac{2 z}{r}} \sum_{k=0}^{\infty}\left(\frac{1}{\beta}\right)^{k} I_{k}\left(\frac{2 z}{r} \beta\right)-e^{-\frac{2 z}{r} I_{0}\left(\frac{2 z}{r} \beta\right)} \\
& \underline{\underline{\underline{(j)}}} 2 e^{-\frac{z}{\mu}}-2 e^{-\frac{2 z}{r}} \sum_{k=0}^{\infty}\left(\frac{1}{\beta}\right)^{k} \sum_{m=0}^{\infty} \frac{1}{m ! \Gamma(m+k+1)}\left(\frac{z}{r} \beta\right)^{2 m+k}-e^{-\frac{2 z}{r}} \sum_{m=0}^{\infty} \frac{1}{m ! \Gamma(m+1)}\left(\frac{z}{r} \beta\right)^{2 m}
\end{aligned}
$$

Where, $(h)$ follows from (17). Then, $(i)$ and $(j)$ are obtained according to

$$
\xi(x, y)=1-e^{-\frac{x^{2}+y^{2}}{2}} \sum_{k=0}^{\infty}\left(\frac{x}{y}\right)^{k} I_{k}(x y)
$$

and the definition of the modified Bessel function of the $k$-th order $I_{k}(x)=\sum_{m=0}^{\infty} \frac{1}{m ! \Gamma(m+k+1)}\left(\frac{x}{2}\right)^{2 m+k}$. Also, $\Gamma(x)=\int_{0}^{\infty} t^{x-1} e^{-t} \mathrm{~d} t$ denotes the Gamma function. In this way, using (23), it can be written

$$
\begin{aligned}
& \int_{\lambda^{*}}^{\infty} \frac{1-F_{Z}(z)}{z^{n}} \mathrm{~d} z \underline{(k)} 2 \int_{\lambda^{*}}^{\infty} z^{-n} e^{-\frac{z}{\mu}} \mathrm{d} z \\
& -2 \sum_{m=0}^{\infty} \sum_{k=0}^{\infty} \frac{1}{m !(m+k) !} \beta^{-2 m} r^{-(2 m+k)} \int_{\lambda^{*}}^{\infty} z^{2 m+k-n} e^{-\frac{2 z}{r}} \mathrm{~d} z \\
& -\sum_{m=0}^{\infty} \frac{1}{(m !)^{2}}\left(\frac{\beta}{r}\right)^{2 m} \int_{\lambda^{*}}^{\infty} z^{2 m-n} e^{-\frac{2 z}{r}} \mathrm{~d} z \\
& \underline{\underline{(l)}} 2 \Gamma\left(1-n, \frac{\lambda^{*}}{\mu}\right) \\
& -2 \sum_{m=0}^{\infty} \sum_{k=0}^{\infty} \frac{\beta^{-2 m} r^{-(2 m+k)}}{m !(m+k) !}\left(\frac{r}{2}\right)^{2 m+k+1-n} \Gamma\left(2 m+k+1-n, \frac{2 \lambda^{*}}{r}\right) \\
& -\sum_{m=0}^{\infty} \frac{1}{(m !)^{2}}\left(\frac{\beta}{r}\right)^{2 m}\left(\frac{r}{2}\right)^{2 m+1-n} \Gamma\left(2 m+1-n, \frac{2 \lambda^{*}}{r}\right)
\end{aligned}
$$

where $(k)$ follows from the fact that $\Gamma(x)=(x-1)$ ! if $x$ is a positive integer value and $(l)$ is obtained by the definition of the incomplete Gamma function
$\Gamma(a, x)=\int_{x}^{\infty} t^{a-1} e^{-t} \mathrm{~d} t$. Finally, setting $n=2$ and 1 in (25) the Equations (20) and (21) are found, respectively. Here, there are some interesting points to be noted:

- Using (20), it can be easily shown that the waterfilling threshold $\lambda^{*}$ is a decreasing function of the average transmission power constraint $T$. That is, more realizations of the variable $Z$, and correspondingly the channel gains, receive powers as the average transmission power constraint increases. Particularly, $\lambda^{*} \rightarrow 0$ as $T \rightarrow \infty$.

- Assuming independent fading channels, i.e., setting $\beta=0$ in (3), the auxiliary variable cdf is simplified to

$$
F_{Z}(z)=\int_{0}^{z} \int_{0}^{z} f_{G_{1}}(x) f_{G_{2}}(y) \mathrm{d} x \mathrm{~d} y=\left(1-e^{-\frac{z}{\mu}}\right)^{2} .
$$

Therefore, from (20), the Lagrange multiplier $\lambda^{\prime \prime}$ is obtained by

$$
\lambda^{*}=\underset{\lambda}{\arg }\left\{\frac{2 \lambda \operatorname{Ei}\left(\frac{-\lambda}{\mu}\right)-2 \lambda \operatorname{Ei}\left(\frac{-2 \lambda}{\mu}\right)-e^{-\frac{2 \lambda}{\mu}}\left(\mu-2 \mu e^{\frac{\lambda}{\mu}}\right)}{\lambda \mu}=T\right\}
$$

and the channel average rate, i.e., (21), is rephrased as

$$
\bar{R}=\operatorname{Ei}\left(\frac{-2 \lambda^{*}}{\mu}\right)-2 \operatorname{Ei}\left(\frac{-\lambda^{*}}{\mu}\right)
$$

where $\operatorname{Ei}(x)$ is the standard exponential integral function $\operatorname{Ei}(x)=\int_{x}^{\infty} \frac{e^{-u}}{u} \mathrm{~d} u, x \geq 0$.

Finally, extension of the results to arbitrary number of receivers can be found in the Appendix.

\section{Simulation results}

Considering different levels of CSI available at the transmitter, Figure 1 shows the channel average rate versus the average transmission power for different correlation conditions. Here, the results under no-CSI and with perfect CSI in uncorrelated channels condition have been plotted as two lower and upper bounds, respectively. Also, the effect of the channels dependencies on the system data transmission efficiency can be further studied in Figure 2. Here, the correlation gain defined as

$$
K \doteq \frac{\bar{R}}{\left.\bar{R}\right|_{\beta=0}},
$$

which is the ratio of the channel average rate, e.g., (21), and the one for uncorrelated channels, e.g. (28), is demonstrated as a function of the channels correlation. 


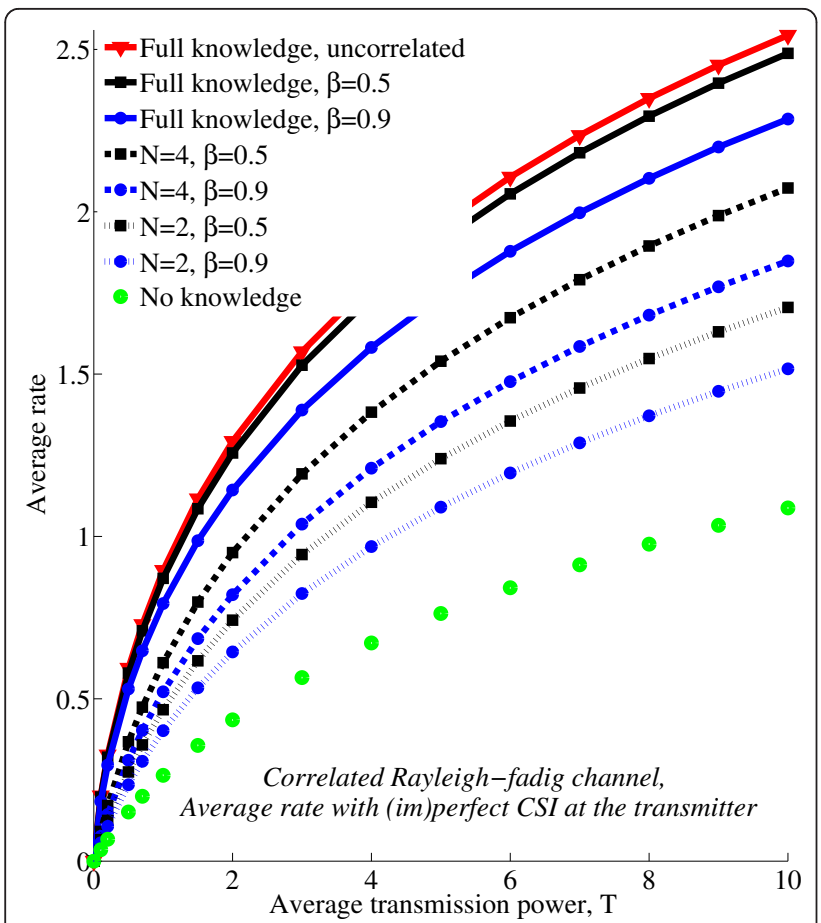

Figure 1 Average rate vs. the average transmission power, correlated Rayleigh-fading channel, $\mu=1$.

Assuming $N=4$ quantization regions, i.e., 2-bits feedback per user, Figure 3 investigates the effect of channels correlation on the optimal quantization boundaries.

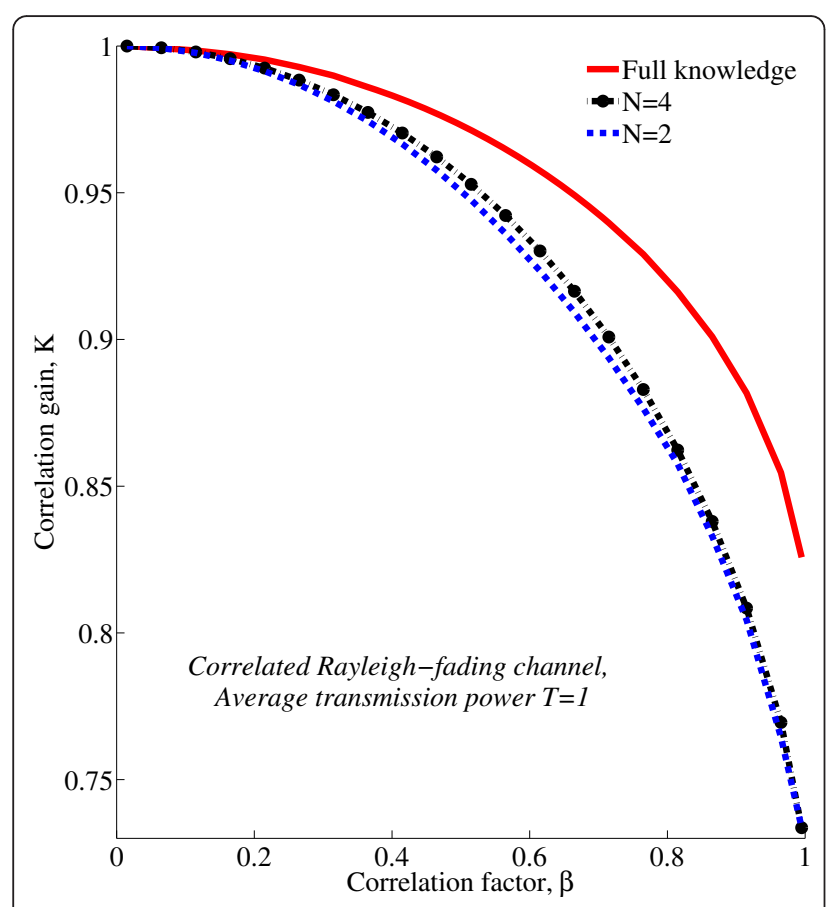

Figure 2 Correlation gain $K$ vs. the correlation factor $\beta$, correlated Rayleigh-fading channel, $T=1, \mu=1$.

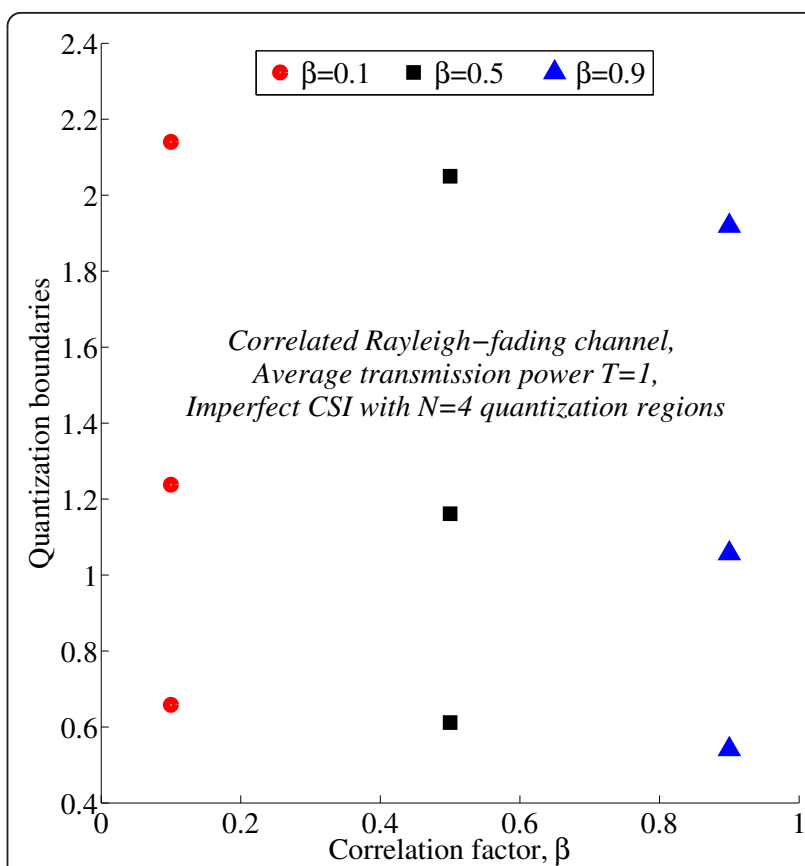

Figure 3 Optimal quantization boundaries for different correlation factors, correlated Rayleigh-fading channel, $T=1, \mu=1$.

Here, the average transmission power is set to $T=1$. Also, Table 1 demonstrates the average rate for different correlation coefficients and number of quantization regions. Finally, Figure 4a,b demonstrate the water-filling threshold, i.e., (20), as a function of the average transmission power $T$ and the correlation factor $\beta$, respectively. Note that the summation terms in (25) converge to zero very fast. Therefore, the water-filling threshold and the average rate can be found accurately with the truncated versions of (25). Also, in all simulations we set the exponential pdfs parameter $\mu=1$.

\subsection{Discussions}

Theoretical and simulation results emphasize a number of interesting points that can be listed as follows:

- For different correlation conditions, considerable performance improvement is achieved via very limited number of feedback bits per user. This point is

Table 1 Channel average rate for different number of quantization regions and correlation coefficients

\begin{tabular}{cccc}
\hline $\boldsymbol{\beta}$ & \multicolumn{3}{c}{$\boldsymbol{N}$} \\
\cline { 2 - 4 } & $\mathbf{2}$ & $\mathbf{3}$ & $\mathbf{4}$ \\
\hline 0 & 0.491 & 0.587 & 0.640 \\
0.1 & 0.490 & 0.586 & 0.639 \\
0.5 & 0.466 & 0.560 & 0.611 \\
0.9 & 0.401 & 0.476 & 0.521 \\
1 & 0.374 & 0.432 & 0.469 \\
\hline
\end{tabular}



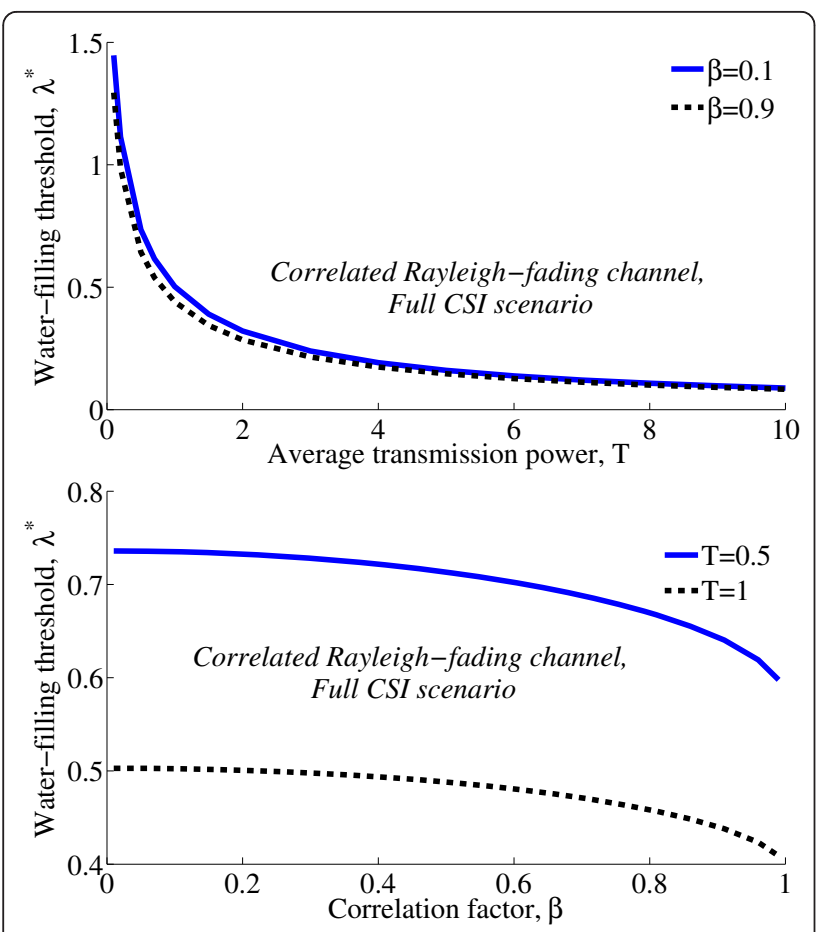

Figure 4 Optimal water-filling threshold as a function of (a) average transmission power constraint $T$ and (b) correlation coefficient $\beta$, correlated Rayleigh-fading channel, $\mu=1$.

useful particularly in networks with a large number of users where the feedback load is an important issue. Moreover, the transmitter CSI is more effective when the channels dependency decreases (Figures 1, 2, and Table 1).

- The effect of scheduling and multiuser diversity reduces with the channels correlation, although the rate reduction is ignorable in low correlation conditions (Figures 1 and 2). There is an interesting intuition behind this point; In a system with a number of users experiencing independent fading conditions it is more likely that, at any time instant, one of the users experiences good channel quality. Therefore, the data transmission efficiency can be improved by always communicating the best users (multiuser diversity). However, if the channels are not independent, the probability that one of the users has good channel quality while the others experience bad channels, and correspondingly the effect of multiuser diversity, decreases. Therefore, it is expected that for users close to each other, for instance the users in a single cell, e.g., [25-28]], the practical gain due to multiuser diversity would be less than the one theoretically obtained under independent channels assumptions. Note that the conclusion is valid for any number of users. Also, it is interesting to mention that, although channels correlation reduces the forward channel data transmission efficiency, it is very helpful for feedback compression of multiuser channels, as discussed in, e.g., [3-5].

- Increasing the channels dependencies, the quantization boundaries converge together (Figure 3). Furthermore, with full correlation between the channels, the results are simplified to the ones obtained for single-user networks.

- The water-filling threshold reduces as the average transmission power or the channels correlation increases (Figure 4). This is intuitively correct because with higher correlations the probability that lower channels gains realizations have the chance of data transmission increases. Therefore, they should receive more power as they have more contribution on the average rate.

Finally, note that the conclusions are valid independent of the fading distributions and the number of receivers.

\section{Conclusion}

This article studies the average rate of multiuser Rayleighfading channels when there is correlation between the users fading channels. The channel average rate is obtained in both perfect and imperfect transmitter CSI conditions under quasi-static channel assumption. Theoretical and simulation results show that substantial performance improvement is achieved with a limited number of feedback bits per user. On the other hand, while average rate reduction due to channels dependencies is ignorable in low correlation conditions, the effect of scheduling and multiuser diversity on the average rate reduces substantially as the fading channels dependency increases. The results are helpful for scheduling in the cases where the users are close to each other, for instance in single-cell networks. Finally, extending the results to the case of cellular networks is an interesting topic which is left for the future.

\section{Appendix 1: Extension of the results to arbitrary number of receivers}

With the same procedure as in Section 3, the system average rate in the presence of $M$ users experiencing symmetric correlated fading distributions and using quantization function (6) is obtained as

$$
\begin{aligned}
& \bar{R}=\sum_{i=1}^{N} \Omega(M, i) \log \left(1+\hat{g}_{i} T_{i}\right), \\
& \Omega(M, i)=\sum_{\forall j_{1} \ldots j_{i}}\left(\begin{array}{c}
M \\
1
\end{array}\right)\left(\begin{array}{c}
M-1 \\
j_{1} \ldots j_{i}
\end{array}\right) \frac{P_{j_{1} \ldots j_{i}}}{j_{i}+1}, \\
& \sum_{w=1}^{i} j_{w}=M-1
\end{aligned}
$$

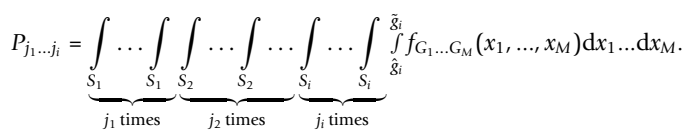


Here, $\left(\begin{array}{c}M \\ 1\end{array}\right)$ is the " $n$ choose $k$ " operator, $\left(\begin{array}{l}M-1 \\ j_{1} \ldots j_{i}\end{array}\right)=\frac{(M-1) !}{j_{1} ! \ldots j_{i} !}$ and $f_{G_{1} \ldots G_{M}}$ is the correlated channels fading pdf. Moreover, $\Omega(M, i)$ is the probability that, $j_{w}, w=1, \ldots, i$, of the $M-1$ unscheduled users are in the quantization region $S_{w}$, the scheduled user which can be any of the $M$ users is in the quantization region $S_{i}$, one of the $\left(j_{i}+1\right)$ users in the region $S_{i}$ is selected randomly (with probability $\frac{1}{j_{i}+1}$ ) and the selected user channel gain supports the rate, e.g., $G_{1} \in\left[\hat{g}_{i}, \tilde{g}_{i}\right)$. Also, the average transmission power, i.e., (10), is rephrased as

$$
\begin{aligned}
& \bar{T}=\sum_{i=1}^{N} \Omega^{\prime}(M, i) T_{i}, \\
& \Omega^{\prime}(M, i)=\Omega(M, i) \frac{P_{j_{1} \cdot j_{i}}^{P_{1}}}{P_{j_{1}, j_{i}}}
\end{aligned}
$$

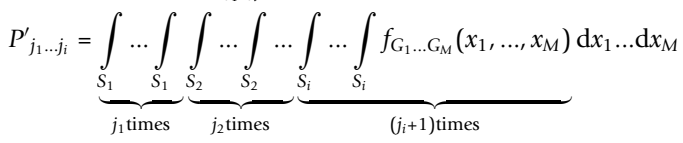

where $P_{j_{1} \ldots j_{i}}^{\prime}$ is the probability that there are $j_{w}, w=1, \ldots$, $i$ - 1 , gains in the quantization region $S_{w}$ and $\left(j_{i}+1\right)$ gains in the region $S_{i}$. Replacing (30) and (31) in (13) the power-limited average rate optimization problem can be solved based on the channels distributions. Assuming perfect CSI available at the transmitter, on the other hand, the average rate is obtained by (18) in which the auxiliary parameter $Z$ is redefined as $Z=\max \left(G_{1}, \ldots, G_{M}\right)$.

\section{Endnotes}

${ }^{a}$ The noise parameter $Z_{k, t}$ represents the Gaussian interferences received from the other users/cells as well. ${ }^{\mathrm{b}} \mathrm{As}$ discussed in [43], the information theoretic results of quasi-static fading channels match the results of actual codes for practical code lengths, e.g., $L_{\mathrm{c}} \simeq 100$ channel uses. 'In an AWGN channel with constant gain $g$ and transmission power $T$, the maximum rate is obtained by $\log (1+g T)$ [41]. This is particularly because, as there is perfect CSI at the receiver, likelihood decoding can be successfully implemented at the receiver.

\section{Algorithm 1 Average rate optimization}

I. For a given power constraint $T$, consider $J$, e.g. $J=20$, randomly generated vectors $\Lambda^{j}=\left[\hat{g}_{1}, \ldots, \hat{g}_{N}, \tilde{g}_{0}, \tilde{g}_{1}, \ldots, \tilde{g}_{N}\right]$ such that $\tilde{g}_{i-1} \leq \hat{g}_{i}<\tilde{g}_{i}, \tilde{g}_{0}=0, \hat{g}_{N}=\infty$.

II. For each vector, do the following procedures

1) Determine the the probability terms of (7) and (10) based on (15).
2) Determine the average rate according to (7) and (14).

III. Find the vector which results in the highest average rate, i.e., $\Lambda^{i}$ where $\bar{R}^{j} \leq \bar{R}^{i}, \forall_{j}=1, \ldots, J$.

IV. $\Lambda^{1} \leftarrow \Lambda^{i}$.

V. Generate $b \ll J$, e.g., $b=5$, vectors $\Delta^{j, \text { new }}, j=1, \ldots, b$ around $\Lambda^{1}$. These vectors should also satisfy the constraints introduced in I.

VI. $\Lambda^{j+1} \leftarrow \Lambda^{j, \text { new }}, j=1, \ldots, b$.

VII. Regenerate the remaining vectors $\Lambda^{j}, j=b+2, \ldots, J \quad$ randomly such that $\Lambda^{j}=\left[\hat{g}_{1}, \ldots, \hat{g}_{N}, \tilde{g}_{0}, \tilde{g}_{1}, \ldots, \tilde{g}_{N}\right] \quad$ and $\tilde{g}_{i-1} \leq \hat{g}_{i}<\tilde{g}_{i}, \tilde{g}_{0}=0, \hat{g}_{N}=\infty$.

VII. Go to II and continue until convergence.

\section{Competing interests}

The authors declare that they have no competing interests.

Received: 8 July 2011 Accepted: 8 February 2012

Published: 8 February 2012

\section{References}

1. M Torabi, D Haccoun, Performance analysis of joint user scheduling and antenna selection over MIMO fading channels. IEEE Signal Process Lett. 18(4), 235-238 (2011)

2. L Jin, X Gu, Z Hu, Low-complexity scheduling strategy for wireless multiuser multiple-input multiple-output downlink system. IET Commun. 5(7), 990-995 (2011). doi:10.1049/iet-com.2010.0358

3. B Makki, T Eriksson, Efficient channel quality feedback signaling using transform coding and bit allocation, in Vehicular Technology Conference, VTC, Ottawa, ON, pp. 1-5 (2010)

4. T Eriksson, $\mathrm{T}$ Ottosson, Compression of feedback for adaptive transmission and scheduling. Proc IEEE. 95(12), 2314-2321 (2007)

5. T Eriksson, $\mathrm{T}$ Ottosson, Compression of feedback in adaptive OFDM-based systems using scheduling. IEEE Commun Lett. 11(11), 859-861 (2007)

6. P Viswanath, DNC Tse, R Laroia, Opportunistic beamforming using dumb antennas. IEEE Trans Inf Theory. 48(6), 1277-1294 (2002). doi:10.1109/ TIT.2002.1003822

7. G Dimic, ND Sidiropoulos, On downlink beamforming with greedy user selection: performance analysis and a simple new algorithm. IEEE Trans Signal Process. 53(10), 3857-3868 (2005)

8. VKN Lau, Proportional fair space-time scheduling for wireless communications. IEEE Trans Commun. 53(8), 1353-1360 (2005). doi:10.1109/ TCOMM.2005.852841

9. M Sharif, B Hassibi, On the capacity of MIMO broadcast channels with partial side information. IEEE Trans Inf Theory. 51(2), 506-522 (2005). doi:10.1109/TIT.2004.840897

10. F Boccardi, F Tosato, G Caire, Precoding schemes for the MIMO-GBC, in Int Zurich Seminar on Commun, Zurich, pp. 10-13 (Feb 2006)

11. W Yu, T Lan, Transmitter optimization for the multi-antenna downlink with per-antenna power constraints. IEEE Trans Signal Process. 55(6), 2646-2660 (2007)

12. A Wiesel, YC Eldar, S Shamai, Zero-forcing precoding and generalized inverses. IEEE Trans Signal Process. 56(9), 4409-4418 (2008)

13. S Sanayei, A Nosratinia, Exploiting multiuser diversity with only 1-bit feedback, in WCNC, vol. 2. New Orleans, LA, pp. 978 (2005)-983

14. K-K Wong, J Chen, Near-optimal power allocation and multiuser scheduling with outage capacity constraints exploiting only channel statistics. IEEE Trans Wirel Commun. 7(3), 812-818 (2008)

15. YS Al-Harthi, AH Tewfik, MS Alouini, Multiuser diversity with quantized feedback. IEEE Trans Wirel Commun. 6(1), 330-337 (2007) 
16. M Kountouris, T SSIzer, D Gesbert, Scheduling for multiuser MIMO downlink channels with ranking-based feedback. Eur J Adv in Signal Process. 2008, 854120 (2008). doi:10.1155/2008/854120

17. H Shirani-Mehr, G Caire, MJ Neely, MIMO downlink scheduling with nonperfect channel state knowledge. IEEE Trans Commun. 58(7), 2055-2066 (2010)

18. H Nam, M-S Alouini, Multiuser switched diversity scheduling systems with per-user threshold. IEEE Trans Commun. 58(5), 1321-1326 (2010)

19. H Shirani-Mehr, H Papadopoulos, SA Ramprashad, G Caire, Joint scheduling and ARQ for MU-MIMO downlink in the presence of inter-cell interference. IEEE Trans Commun. 59(2), 578-589 (2011)

20. WINNER-Wireless-World-Initiative-New-Radio http://projects.celtic-initiative. org/winnert/

21. 3GPP http://www.3gpp.org

22. K Huang, R Heath, J Andrews, Limited feedback beamforming over temporally-correlated channels. IEEE Trans Signal Process. 57(5), 1959-1975 (2009)

23. R1-051334, CQI feedback scheme for EUTRA, Motorola, RAN1 meeting 43, Seoul, Republic of Korea. (2005)

24. R1-062772, Compressed CQI reporting scheme, NEC, RAN WG1 meeting 46, Seoul, Republic of Korea. (2006)

25. N Damji, T Le-Ngoc, Dynamic resource allocation for delay-tolerant services in downlink OFDM wireless cellular systems, in ICC, Beijing, China. 5, 3095-3099 (2005)

26. F Sun, M You, J Liu, Z Shi, P Wen, J Liu, Genetic algorithm based multiuser scheduling for single- and multi-cell systems with successive interference cancellation. in PIMRC, Istanbul, Turkey 1230-1235 (2010)

27. AG Gotsis, NT Koutsokeras, P Constantinou, Radio resource allocation and packet scheduling strategies for single-cell OFDMA packet networks. VTC, Baltimore, MD 1847-1851 (2007)

28. P Marbach, Y Lu, Active queue management and scheduling for wireless networks: the single-cell case. in CISS, Princeton, NJ 1560-1565 (2006)

29. HA Suraweera, PJ Smith, M Shafi, Capacity limits and performance analysis of cognitive radio with imperfect channel knowledge. IEEE Trans Veh Technol. 59(4), 1811-1822 (2010)

30. KS Ahn, RW Heath, Performance analysis of maximum ratio combining with imperfect channel estimation in the presence of cochannel interferences. IEEE Trans Wirel Commun. 8(3), 1080-1085 (2009)

31. Q Sun, DC Cox, HC Huang, A Lozano, Estimation of continuous flat fading MIMO channels. IEEE Trans Wirel Commun. 1(4), 549-553 (2002). doi:10.1109/TWC.2002.804178

32. TL Marzetta, BLAST training: estimating channel characteristics for high capacity space-time wireless, in 37th Annual Allerton Conference on Communication, Control and Computing, vol. 1. (Allerton House, Monticello, IL, 1999)(4), , pp. 958-966

33. C Tellambura, ADS Jayalath, Generation of bivariate rayleigh and nakagamiM fading envelopes. IEEE Commun Lett. 4(5), 170-172 (2000). doi:10.1109/ 4234.846501

34. B Makki, T Eriksson, Data transmission in the presence of noisy channel state feedback and outage probability constraint, in International Symposium on Information Theory and its Applications, ISITA, Taichung, Taiwan, pp. 458-463 (2010)

35. B Makki, T Eriksson, On the average rate of quasi-static fading channels with ARQ and CSI feedback. IEEE Commun Lett. 14(9), 806-808 (2010)

36. TT Kim, M Skoglund, On the expected rate of slowly fading channels with quantized side information. IEEE Trans Commun. 55(4), 820-829 (2007)

37. S Ekbatani, F Etemadi, H Jafarkhani, Throughput maximization over slowly fading channels using quantized and erroneous feedback. IEEE Trans Commun. 57(9), 2528-2533 (2009)

38. S Ekbatani, F Etemadi, $\mathrm{H}$ Jafarkhani, Outage behavior of slow fading channels with power control using partial and erroneous CSIT. IEEE Trans Inf Theory. 56(12), 6097-6102 (2010)

39. JG Proakis, Digital Communications, 4th edn. (McGraw Hill, New York, 2001)

40. B Sklar, Rayleigh fading channels in mobile digital communication systems. I characterization IEEE Commun Mag. 35(7), 90-100 (1997)

41. TM Cover, JA Thomas, Elements of Information Theory (Wiley, New York, 1992)

42. B Makki, M Noori Hosseini, SA Seyyedsalehi, N Sadati, Unaligned training for voice conversion based on a local nonlinear principal component analysis approach. Neural Comput Appl. 19(3), 437-444 (2009)
43. G Caire, G Taricco, E Biglieri, Optimum power control over fading channels. IEEE Trans Inf Theory. 45(5), 1468-1489 (1999). doi:10.1109/18.771147

doi:10.1186/1687-1499-2012-38

Cite this article as: Makki and Eriksson: Multiuser diversity in correlated Rayleigh-fading channels. EURASIP Journal on Wireless Communications and Networking 2012 2012:38.

\section{Submit your manuscript to a SpringerOpen ${ }^{\mathcal{O}}$ journal and benefit from:}

- Convenient online submission

- Rigorous peer review

- Immediate publication on acceptance

- Open access: articles freely available online

- High visibility within the field

- Retaining the copyright to your article

Submit your next manuscript at $\boldsymbol{~ s p r i n g e r o p e n . c o m ~}$ 